H. Bohr, Fastperiodische Funktionen. (Ergebnisse der Mathematik und ihrer Grenzgebiete, Bd. 5.) J. Springer, Berlin 1938. Preis geh. RM 11,40.

Das varliegende Buch ist eine Ausarbeitung der meisterhaften Vorlesungen, die Verfasser an mehreren Universitäten gehalten hat. Der einleitende erste Teil enthält eine (auf die Verallgemeinerung durch die fastperiodischen Funktionen zugeschnittene) Darstellung der reinperiodischen Funktionen und ihrer Fourierreihen. Der Inhalt des Hauptteiles, der zunächst neben den Definitionen der fastperiodischen (reellen, stetigen) Funktionen und ihrer Fourierreihen die einfachsten Eigenschaiten und Rechenregeln derselben bringt, gruppiert sich vor allem um zwei Sätze: um den Satz von der eindeutigen Bestimmtheit jeder fastperiodischen Funktion durch ihre Fourierreihe (er ist mit der Parsevalschen Gleichung äquivalent) and den Hauptsatz, daß die fastperiodischen Funktionen mit den durch endliche Summen $\Sigma_{a_{n}} e^{i \lambda_{n} x^{x}}$ gleichmäßig approximierbaren Funktionen identisch sind. Ein erster Anhang unterrichtet über die Verallgemeinerung der fastperiodischen Funktionen durch Stepanoff, Besicovitch und Weyl; ein zweiter über die fastperiodischen analytischen Funktionen einer komplexen Variablen und ihre Dirichletschen Reihen. - Das Büchlein, das jedem Leser einen hohen Genuß bereitet, bietet eine vorbildliche Einführung in die Theorie.

\title{
G. Nöbeling.
}

E. J. Berg, Rechnung mit Operatoren, nach Oliver Heaviside, ihre Anwendung in. Technik und Physik. Deutsche Bearbeitung von 0 . G r a m is ch und H. Tropper. 188 Seiten. R. Oldenbourg, München und Berlin 1932. Preis geh, RM 10,-, geb. RM 12,--.

Symbolische Verfahren nehmen jederzeit die Aufmerksamkeit des Mathematikers in eigenartiger Weise gefangen. Ihr Vorteil liegt in der raschen Gewinnung gewisser Ergebnisse; dem steht gegenüber, daß die zulässigen Operationen jedesmal in besonderer Weise eingeschränkt sind. Der Ablauf der Entwicklung ist am leichtesten zu übersehen, wenn man weit zurückgreift, etwa das Rechnen mit komplexen Zahlen betrachtet, dessen Ergiebigkeit auch an Ergebnissen im reellen Gebiet seinerzeit dasselbe Gemisch von Befriedigung und Mißtrauen ausgelöst hat, wie heute neuere symbolische Rechnungsweisen. Das Mißtrauen ist geschwunden, seitdem genau festgestellt wurde, welche Aussagen iuber reelle Zahlen im komplexen Gebiet erhalten bleiben. Demnach wird man also ein symbolisches Rechenverfahren als wertvoll ansehen, wenn es eine genügende Ausbeute an Ergebnissen liefert und die Rechenregeln, die für die Symbole aus der gewöhnlichen Rechnung übernommen werden dürfen, bekannt sind, wobei offenbar die Anzahl dieser Rechenregeln nicht gar zu gering sein darf.

Daß die Heavisideschen Operatoren eine Fülle von Ergebnissen liefern, wird durch ihre übersichtliche Zusammenstellung in dem vorliegenden Werk von nevem bewiesen. Wenn auch die elektrotechnischen Aufgaben überwiegen, so kommen doch auch die Anwendungen auf Mechanik und andere Gebiete der Physik zur Geltung. Der zweiten vorhin ausgesprochenen Forderung ist wohl nicht in dem Maße entsprochen, wie es der Mathematiker wünschen würde, insbesondere sind die Fälle, in denen die Symbole den gewöhnlichen Rechenregeln nicht folgen, mehr im Vorbeigehen erwähnt worden, so daß wohl der Wunsch nach einer schärferen Feststellung der Eigenschaften der Operatoren offen bleibt. Der Vergleich mit den komplexen Zahlen zeigt jedoch, daß diese zweite Forderung sich auch sonst erst im Laufe der Zeit durchgesetzt hat, so daß man hoffen darf, daß auch für die Heavisidesche Operatorenrechnung die strenge Begründung in nicht zu ferner Zeit nachgetragen werden wird.

$Z u$ erwähnen wäre noch, daß in zwei Schlußkapitein eine sehr eingehende Darstellung der Graeffeschen Aullösungsmethode algebraischer Gleichungen und eine Zusammenstellung der Formeln und in einem Anhang eine Uvbersicht über das Lebenswerk Heavisides angeschlossen ist, während schon im Vorwort einiges über seine eigenartige Persönlichkeit gesagt ist.

L. Schrutka.

A. Henry, Le calcul des différences finies et ses applications avee de nombreux exemples, traités dans tous leurs détails et un grand nombre d'exereices 